\title{
Response to fluorescein angiography and OCT
}

\author{
Roberto Gallego-Pinazo • M. Díaz-Llopis • R. Dolz • \\ S. Martínez-Castillo
}

Received: 9 July 2010 / Accepted: 12 July 2010 / Published online: 29 July 2010

(C) Springer-Verlag 2010

\section{Dear Editor,}

Dr Gupta confirms that we are living actually in the era of optical coherence tomoghraphy (OCT). We do agree with the point that the OCT reveals static information only, whereas fluorescein angiography (FA) is a dynamic investigation. However, the static information is very considerably more precise with OCT than any clue given by the FA with regard to macular status in retinal vascular disorders.

We routinely use spectral domain OCT (Cirrus HD-OCT, Carl Zeiss Meditec, Inc.) in our daily practice to assess the macular anatomy in the context of retinal vascular diseases. On the other hand, we perform FA only in selected cases, and mostly for investigational or educational purposes. Spectral domain OCT provides high-resolution images of the macula and a retinal thickness map which is superimposed to a retinography, making this perfectly suitable for targeting the thickened areas with laser photocoagulation.

In addition, nowadays is not only the era of OCT, but also the era of the vascular endothelial growth factor (VEGF) intravitreal inhibitors. This therapies have enabled the retinal physicians to improve visual outcomes in patients with age-related macular degeneration, retinal vein occlusions, or diabetic macular edema. But the cost of these medications leads to the need to find the way to reduce the number of injections, and to establish a trend towards a pro re nata individualized treatment. And of course this leads to

R. Gallego-Pinazo $(\bowtie) \cdot$ M. Díaz-Llopis $\cdot$ R. Dolz $\cdot$

S. Martínez-Castillo

Department of Ophthalmology, University Hospital La Fe,

Av Campanar,

2146009 Valencia, Spain

e-mail: robertogallego@comv.es

M. Díaz-Llopis

Faculty of Medicine, University of Valencia,

Valencia, Spain more frequent visits to the Ophthalmology departments. Therefore we need an efficient, safe, and fast image acquisition technique to evaluate these patients. We have no doubt that spectral domain OCT is this technique.

Focusing on diabetic macular edema (DME), we consider the OCT as the standard technique for the quantitative and qualitative evaluation, both in the baseline diagnosis and the follow-up visits. Only the evaluation of ischemic changes is a limiting factor to develop FAindependent evaluations in diabetic patients. However, Yeung et al [1] showed that there is an excellent correlation between OCT findings and retinal ischemia (retinal nerve fibre layer thinning and retinal ganglion cell loss); moreover, when considering the macular perfusion status, we can highly suspect this condition if we find a low visual acuity and a macular vascular network rupture in a current funduscopy. Despite the continuous evolution and improvement of the OCT technology, we agree with Dr Gupta in his statement that FA is still the gold standard procedure to assess the presence of macular ischemia.

In conclusion, we may routinely plan an initial macular laser photocoagulation, as well as further retreatments, with the help of the retinal thickness map provided by spectral domain OCT [2], without the need to perform a timeconsuming, potentially harmful technique such as FA.

\section{References}

1. Yeung L, Lima VC, Garcia P, Landa G, Rosen RB (2009) Correlation between spectral domain optical coherence tomography findings and fluorescein angiography patterns in diabetic macular edema. Ophthalmology 116:1158-1167

2. Gallego-Pinazo R, Díaz-Llopis M, Dolz R, García-Delpech S, Millán-Salvador JM, Arévalo JF (2009) Macular focal treatment guided by the retinal thickness map of the HD-OCT Cirrus versus fluorescein angiography for retinal vein occlusions. Arch Soc Esp Oftalmol 84:597-598 